\title{
Estimates of the Infrared Radiation Temperature Correction for Cylindrical Temperature Sensors ${ }^{1}$
}

\author{
Claude E. Duchon \\ The University of Texas \\ (Manuscript received 15 October 1963, in revised form 13 December 1963)
}

\begin{abstract}
A series of nomograms is presented showing the longwave radiation temperature correction for various thermocouple wire diameters, different values of emitted and absorbed radiation, and for wind speeds from zero to $10 \mathrm{~m} \mathrm{sec}^{-1}$. The temperature correction is computed from the steady-state balance between radiative and convective heat transfers and is to be added to the measured temperature. The radiation field is based upon previous measurements made under clear nighttime skies and the free and forced convection correlation formulae are adopted from wind tunnel experiments.
\end{abstract}

\section{Introduction}

This paper is a sequel to one concerning the radiative temperature correction for spherical temperature elements (Duchon, 1963).

The results of this paper apply to unshielded cylindrical bodies, and, in particular, thermocouples, used in temperature measurement in the lower atmosphere under clear nighttime skies. Thermocouples can be fabricated into cylinders by a technique described by Thornthwaite. ${ }^{2}$

Cylinder, wire, thermocouple and sensor will be used without strict distinction throughout this paper. Generally, cylinders are referred to in theoretical analysis, cylinders and wires are utilized in the experimental heat transfer work, and we shall use the experimental data to estimate the radiative temperature correction for thermocouples used as temperature sensors.

The radiative temperature correction is found for the case of zero wind speed, i.e., pure natural or free convection, and for the case of pure forced convection at various transverse wind speeds, and for both cases at different values of observed air temperature, thermocouple diameter and total incident radiation.

\section{Mathematical development}

Let us assume that thermal equilibrium between the cylindrical element and its environment is everpresent and that radiation and convection are the only relevant heat transfer processes. Following the development shown by Duchon (1963), we arrive immediately at

\footnotetext{
1 The research in this paper has been supported by the Air Force Cambridge Research Laboratories, Office of Aerospace Research, under Contract AF 19(628)-2475.

${ }^{2}$ Thornthwaite, C. W., et al., 1951: Micrometeorology of the surface layer of the atmosphere. The Johns Hopkins University Laboratory of Climatology, Interim Report No. 13, p. 4.
}

what may be designated the general infrared radiation temperature correction equation in the form

$$
T_{a}-T_{c}=\frac{d}{k S}\left(\epsilon S \sigma T_{c}^{4}-a_{1} \frac{d E}{d t} \downarrow-a_{2} \frac{d E}{d t} \uparrow\right) / \mathrm{Nu},
$$

where the notation is

$$
T_{a}=\text { air temperature }
$$

$T_{c}=$ cylinder, wire, thermocouple, and sensor temperature

$S=$ surface area of a cylinder

$d=$ cylinder diameter

$k=$ thermal conductivity of air

$h=$ average surface coefficient of convective heat transfer

$\mathrm{Nu}=h d / k=$ Nusselt number

$d E / d t \downarrow, d E / d t \uparrow=$ the rates of infrared energy interception by the sensor from above and below its horizon, respectively

$a_{1}, a_{2}=$ the absorptivities of the sensor for downward and upward longwave radiation, respectively

$\epsilon=$ emissivity of the sensor

$\sigma=$ Stefan-Boltzmann constant.

As they are employed in $\mathrm{Eq}$ (1), the absorptivities and emissivity are weighted means; weighted, respectively, with the spectral distribution of the incident energy and the blackbody energy of the sensor.

The terms inside the parenthesis in $\mathrm{Eq}$ (1) comprise the radiation energy balance of the cylinder. Now in practice, the two components of radiant energy namely, $(d E / d t) \downarrow$ and $(d E / d t) \uparrow$, are not measurable. However, we can relate these quantities to the downward and upward flux densities of radiation detected by a flat plate type of themal radiometer. 
As the first step toward this goal, we seek relationships for the spatial distribution of the intensity of sky and ground radiation. For the downward intensity of radiation under clear nighttime skies we shall employ the empirical formulation suggested by Duchon (1963), viz.,

$$
I \downarrow(\theta) / B=1-c_{1} \exp \left(-c_{2} \sec ^{\frac{1}{2}} \theta\right),
$$

where $I \downarrow(\theta)$ is the intensity of radiation, $B$ is the black body intensity of the horizon, $\theta$ is the zenith angle, and $c_{1}$ and $c_{2}$ are parameters. that

For the upward intensity of radiation we shall assume

$$
I \uparrow(\theta)=B .
$$

This assumption seems plausible, at least at low heights, since the radiation from both the horizon and Earth's surface approach that of a blackbody. As their temperature difference increases the error will also increase.

For the second step we can find the radiation impinging on a cylinder and on a plate by integrating $\mathrm{Eq}_{\mathrm{q}}(2)$ and (3) over their associated hemispheres. Finally, we can determine the ratio of the flux density on a cylinder to that on a plate for the downward and upward components of radiation. The mechanics of the integration and the evaluation of the ratios are discussed in the Appendix. Here we shall use only the results.

The expression of this ratio for downward radiation and its average value is

$$
\left(\frac{1}{S / \pi} \frac{d E}{d t} \downarrow\right)_{c} /\left(\frac{1 d E}{A} \frac{d}{d t} \downarrow\right)_{p}=1.03(\pi / 2),
$$

where $S / \pi$ is the longitudinal cross-sectional area of the cylinder, $A$ is the area of the upper surface of the radiometer, and the subscripts $c$ and $p$ refer to a cylinder and a plate, respectively.

The expression for the upward radiation and its value is

$$
\left(\frac{1}{S / \pi} \frac{d E}{d t} \uparrow\right)_{c} /\left(\frac{1}{A} \frac{d E}{d t} \uparrow\right)_{p}=\pi / 2
$$

Upon substituting Eq (4) and (5) into (1) we obtain

$$
T_{a}-T_{c}=\frac{d}{2 k}\left(2 \epsilon \sigma T_{c}^{4}-1.03 a_{1} F \downarrow-a_{2} F \uparrow\right) / \mathrm{Nu},
$$

where $F \downarrow$ and $F \uparrow$ are the flux densities of radiation as detected by a radiometer.

In a later section we shall make recourse to $\mathrm{Eq}(6)$ in order to compute the radiative temperature correction.

\section{Pure free convection formulae}

The amount of heat transferred to or from a cylinder can be obtained by relating the Nusselt number to the
Grashof number where the latter climensionless number may be interpreted as the ratio of the product of the bouyancy and inertia forces to the square of the viscous forces.

Because the range of the Grashof number in this paper varied over more than seven orders of magnitude, it was found necessary to consider two empirical formulae.

The first formula is the semi-logarithmic relation

$$
\mathrm{Nu}^{-1}=0.88-0.43 \log _{10} \mathrm{Gr}
$$

correlated by Collis and William; (1959) who suggest that it is satisfactory over the range $10^{-10}<\mathrm{Gr}<10^{-2}$, where $\mathrm{Gr}$ denotes the Grashof number. The Grashof number is written as $g d^{3} \beta \Delta T \nu^{-2}$ where $g$ is the acceleration due to gravity, $\beta$ is the thermal expansion coefficient of air, $\nu$ is the kinematic viscosity, and $\Delta T$ is the temperature difference betwee $a$ the cylinder and the undisturbed air. In $\mathrm{Eq}$ (7) the Nusselt number was evaluated at the mean value of the temperature of the wire and the undisturbed air; anc the Grashof number at the undisturbed air temperatu:e. This is in contrast to the usual procedure of evaluating the physical properties of the fluid in both dimensi nnless numbers at the mean temperature (McAdams, 1954, p. 176; Gröber, 1961, p. 320). Collis and Williaras ${ }^{3}$ (p. 13) propound that their method is consistent with the physical picture of heat transfer at small Grashof numbers.

For $\mathrm{Gr}>10^{-2}$ we shall make use of the relationship proposed by van der Hegge Zijnen (1957), namely,

$$
\mathrm{Nu}=0.35+0.24 \mathrm{Gr}^{1 / 8} \cdot+0.41 \mathrm{Gr}^{\frac{1}{4}},
$$

in which the dimensionless numb rs were evaluated by the usual procedure previously indicated.

In Section $5 \mathrm{Eq}(7)$ and (8) are incorporated into $\mathrm{Eq}(6)$ for the computation of the radiation temperature correction for free convection.

\section{Pure forced convection}

A large body of heat transfer data is available for cylinders or wires. Much of the not too recent data has been gathered and presented by McAdams (1954, p. 259). However, few data are available for small Reynolds numbers. The Reynolds number is a measure of the ratio of the inertia forces to the viscous forces and is written $v d \nu^{-1}$ where $v$ denotes air speed. For $\operatorname{Re}<44$ we shall make use of the correlation formula determined by Collis and Williams (1959) while for $\operatorname{Re}>44$ we shall utilize the McAdams recommended formula. The transition of $\mathrm{Nu}$ versus Re from the Collis and Williams relation to the McAdams formula is very smooth.

Hence, in the range $\operatorname{Re}<44$ we find

$$
\mathrm{Nu}=0.24+0.56 \mathrm{Re}^{0.45}
$$

${ }^{3}$ Collis, D. C., and M. J. Williams, 1954: Free convection of heat from fine wires. Aero. Res. Lab, Melbourne, Aust., Aero Note 140 (available from U. S. Weather Bureau, Washington
25, D. C.). 
and for $\operatorname{Re}>44$

$$
\mathrm{Nu}=0.32+0.43 \mathrm{Re}^{0.52} .
$$

It was pointed out in the introduction that we seek the variation in radiation temperature correction in passing from zero wind speed through very low to high wind speeds. Accordingly, speeds of 1, 10, 100, and $1000 \mathrm{~cm} \mathrm{sec}^{-1}$ have been chosen. At the lowest speed, where the temperature correction is large, free convection effects arise. A criterion for the onset of bouyancy effects has been proposed by Collis and Williams (1959) on the assumption that there is no deviation from the forced convection regime for low Reynolds numbers until the Reynolds number is reduced to the point where the forced convection Nusselt number equals that due to free convection.

The equation expressing this criterion is

$$
\operatorname{Re}=1.85 \mathrm{Gr}^{0.39},
$$

and its application will be discussed in the next section.

\section{Results}

By alternately substituting Eq (7), (8), (9), and (10) into (6), we have computed the temperature correction $\left(T_{a}-T_{c}\right)$ as a function of the observed sensor temperature $T_{c}$, sensor diameter $d$, wind speed $v$, and total incident radiation $F_{t}$, where $F_{t}=1.03 F \downarrow+F \uparrow$. In the computations we let $\epsilon=a_{1}=a_{2}=1$, which means the sensor is a black body. All computations in this paper were carried out on the CDC 1604 digital computer. Values of the fluid properties used were $k=0.00006 \mathrm{cal} \mathrm{sec}^{-1}$ $\mathrm{cm}^{-1} \mathrm{~K}^{-1}, \beta=0.00367 \mathrm{~K}^{-1}$ and $\nu=0.15 \mathrm{~cm}^{2} \mathrm{sec}^{-1}$.

Fig. 1 shows a plot of the free convection radiation temperature correction for various thermocouple wire diameters and the difference between the outgoing and incoming flux densities $\left(2 \sigma T_{c}{ }^{4}-F_{t}\right)$. The values shown for $\left(2 \sigma T_{c}{ }^{4}-F_{t}\right)$ apply to the range in $T_{c}$ from 270 to $300 \mathrm{~K}$ and in $F_{t}$ from 0.009 to $0.020 \mathrm{cal} \mathrm{sec}^{-1} \mathrm{~cm}^{-2}$.

In Fig. 1 we note the discontinuity in the temperature correction curves in passing from the Collis and Williams equation to the van der Hegge Zijnen equation. In Section 3 it was pointed out that the former authors evaluated the physical properties of the fluid represented in the Grashof number at the undisturbed air temperature whereas the latter author evaluated them at the mean temperature. If a simple example were to be carried out wherein the wire temperature is greater than that of the undisturbed air temperature, the Grashof number evaluated by the former method would be greater than the Grashof number evaluated by the latter. method due only to the difference in the value of kinematic viscosity. Consequently, this would lead to different functional relationships for $\mathrm{Nu}$ versus $\mathrm{Gr}$ around $\mathrm{Gr}=10^{-2}$ and may account for the discontinuity in the temperature correction curves.
A further consequence of the dissimilarity in computing the kinematic viscosity is that with the van der Hegge Zijnen equation no difference occurs in heat transfer whether the thermocouple wire is warmer or cooler than its surroundings whereas in the Collis and Williams equation a disparity does arise.

Figs. 2a, b, c, and d apply to the forced convection regime with wind speeds of $1,10,100$, and $1000 \mathrm{~cm}$ $\mathrm{sec}^{-1}$, respectively. The wind speed for Fig. $2 \mathrm{a}(1 \mathrm{~cm}$ $\mathrm{sec}^{-1}$ ) was sufficiently low so that it was necessary to implement the buoyancy criterion, Eq (11). This was accomplished with the following procedure. The temperature correction was computed from $\mathrm{Eq}_{\mathrm{q}}(6)$ and (9). Then the Grashof number was calculated using this temperature correction. Finally, if the Reynolds number from $\mathrm{Eq}$ (11) was greater than the Reynolds number in $\mathrm{Eq}(9)$, buoyancy effects were in evidence and the computed $\left(T_{a}-T_{c}\right)$ was disregarded. If the former Reynolds number was less than the latter buoyancy effects were nil and the computed $\left(T_{a}-T_{c}\right)$ was accepted. We observe in Fig. 2a that the region of buoyancy effects coincides with the larger values of temperature correction, diameter, and $\left(2 \sigma T_{c}^{4}-F_{\imath}\right)$. This is consistent with theory.

Figs. 1 and 2 represent estimates of the maximum possible radiative temperature correction in that the emissivity and absorptivities were assumed equal to one. To find the temperature correction for values other than one, it will be necessary, and, indeed, just as practical, to let $\epsilon=a_{1}=a_{2}$. This means the sensor acts as a gray body. This being done, we see from Eq (6) that we can multiply $\left(2 \sigma T_{c}{ }^{4}-F_{t}\right)$ by the known or estimated value of emissivity or absorptivity.

Heretofore we have considered only transverse flow, i.e., air flow normal to the axis of the thermocouple. Heat transfer from yawed cylinders has been examined by Baldwin et al. (1960), among others. It is found that heat transfer data correlate satisfactorily when the free stream air speed is multiplied by the sine of the angle of yaw, except when this angle is less than about 20 degrees. In the atmosphere, of course, the wind direction is always variable. To correctly account for the effect of a variable wind direction on the temperature correction in the practical situation becomes problematical. Best results will be obtained when the themocouple is aligned normal to the average wind direction.

To get an idea of the sensitivity of the temperature correction to the measured temperature, we find from the forced convection nomograms that for a change in $T_{c}$ of $\pm 1 \mathrm{C}$ the maximum absolute error in the temperature correction is about $\pm 0.02 \mathrm{deg}$ for $v=1000 \mathrm{~cm}$ $\mathrm{sec}^{-1}$ and about $\pm 0.2 \mathrm{deg}$ for $v=10 \mathrm{~cm} \mathrm{sec}^{-1}$. At any given diameter and for all wind speeds the corresponding percentage error in $\left(T_{a}-T_{c}\right)$ increases from approximately \pm 3 per cent at the larger temperature corrections to greater than 75 per cent at the smaller temperature corrections. For a sensor absorptivity or emissivity 


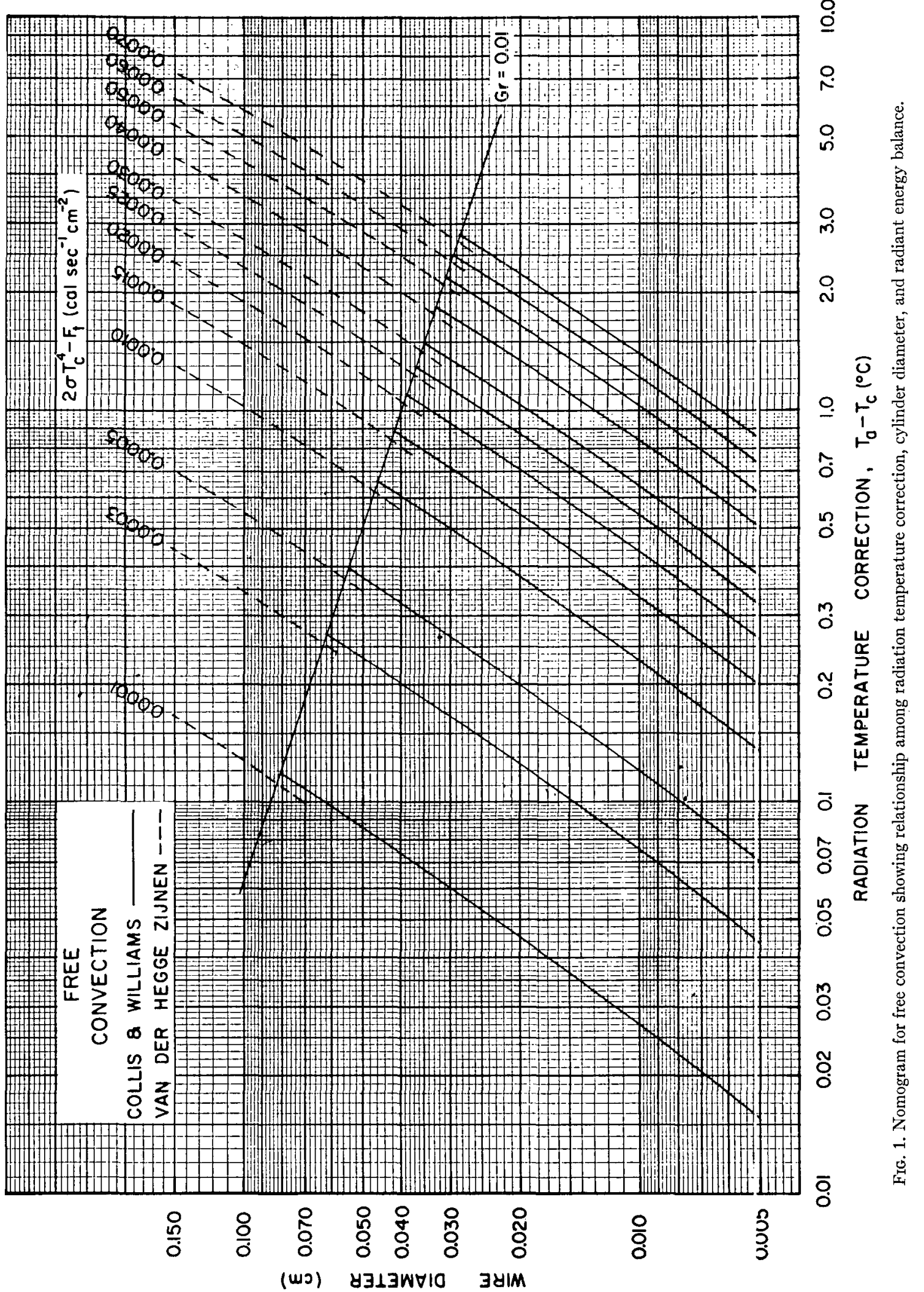



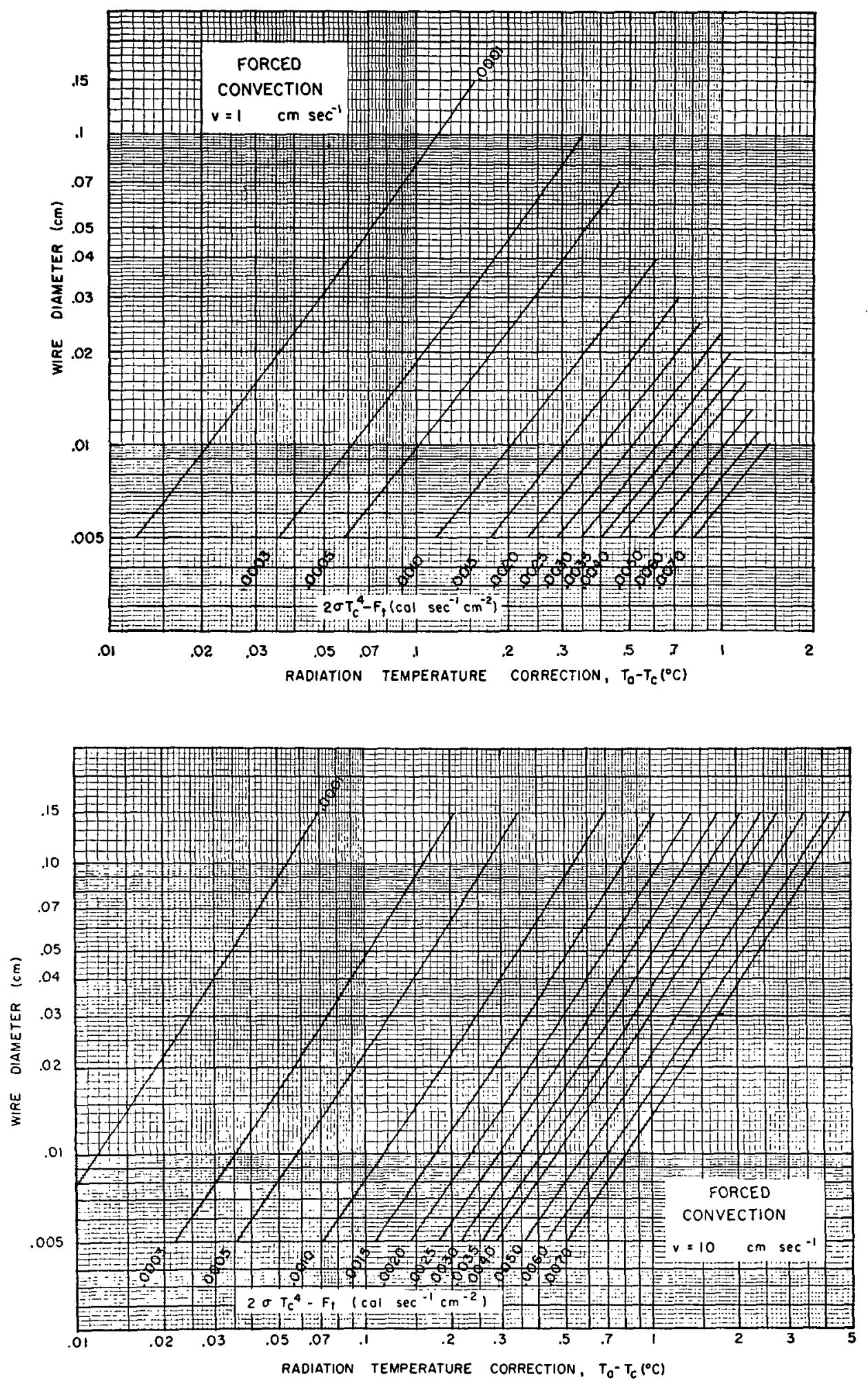

FIG. 2. Nomograms for forced convection showing relationship among radiation temperature correction, cylinder diameter, and radiant energy balance for various wind speeds. 

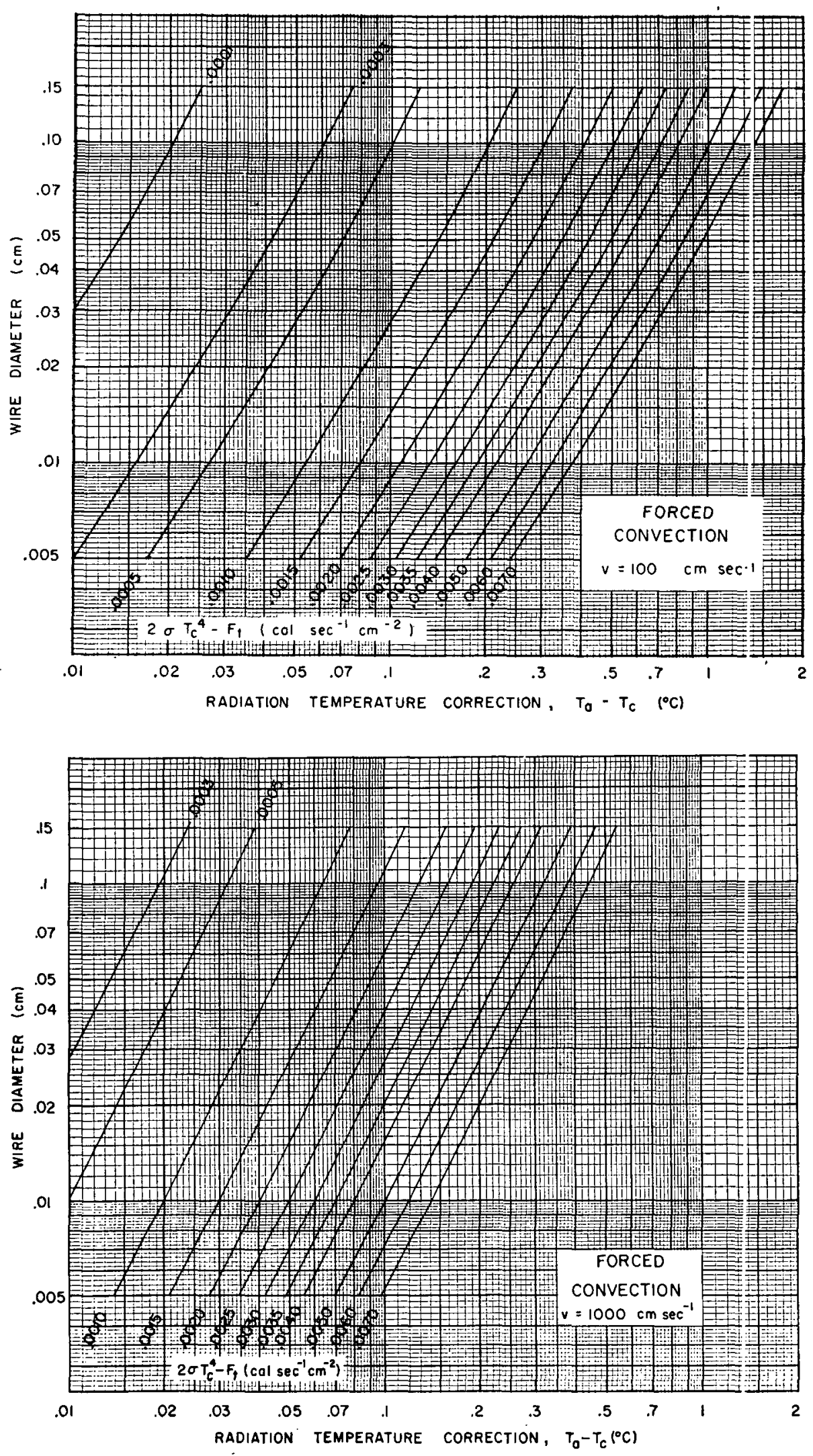

FIG. 2. Continued. 
less than one, both the absolute and percentage error will decrease.

\section{Example}

Let us consider a simple example that will demonstrate the procedure in arriving at the temperature correction. Assume that we are using a horizontally positioned thermocouple whose exposed length is sufficiently large that two-dimensional heat transfer occurs. The thermocouple is located about a meter above the surface and its diameter is $0.04 \mathrm{~cm}$. Over the period of observation the average wind speed was $250 \mathrm{~cm} \mathrm{sec}^{-1}$, the observed temperature $283.5 \mathrm{~K}$, the downward flux density of radiation $6.54 \times 10^{-3}$ and the upward flux density $8.06 \times 10^{-3} \mathrm{cal} \mathrm{sec}^{-1} \mathrm{~cm}^{-2}$. Further, let us say the emissivity (or absorptivity) is 0.5 . Accordingly, we find $\epsilon\left(2 \sigma T_{c}{ }^{4}-F_{t}\right)=0.00133$. On Fig. 2c we find $\left(T_{a}-T_{c}\right)$ $=0.17 \mathrm{C}$ for $\nu=100$ and on Fig. $2 \mathrm{~d}\left(T_{a}-T_{c}\right)=0.056 \mathrm{C}$ for $v=1000 \mathrm{~cm} \mathrm{sec}^{-1}$. With $\log -\log$ interpolation, the desired temperature correction is found to be approximately $0.11 \mathrm{C}$. This then is the estimate of the temperature correction to be added to the observed temperature.

\section{Discussion}

Free convection. When using the results in Fig. 1 it is necessary to keep in mind the significance of the ratio of the length of the exposed wire to its diameter, i.e., the ratio $l / d$.

The magnitude of the end-effects, namely, metallic heat conduction along the wire to the wire supports and the deviation of the coefficient of heat transfer from that for two-dimensional convection are related to the ratio $l / d$.

The metallic heat conduction effect has been investigated by Collis and Williams ${ }^{3}$ for diameters common to hot wire anemometers. Their results for a wire diameter of $0.000295 \mathrm{~cm}$ indicate that the ratio $l / d$ should be about 20,000 in order that this end-effect be negligible.

Although they did not investigate conduction effects for wire diameters in the range employed here they assert that the critical ratio should become less as the diameter increases; i.e., conduction effects become unimportant at lower values of $l / d$ as the diameter increases. If we consider the transfer of heat by conduction and convection in units of power per unit length per unit temperature difference the following argument confirms their assertion. For a constant ratio of $l / d$ the convective transfer is known to increase with an increase in wire diameter while at the same time the transfer by conduction is invariant.

The range in diameter in the present investigation varies from more than one to almost three orders of magnitude greater than the diameter of the wire investigated by Collis and Williams. Therefore, we might expect the ratios of $l / d$ for which conduction can be neglected to be much smaller than 20,000; perhaps 2,000 and less.

The second end-effect, that of the distortion in twodimensional convection, occurs where the wire receives its support and depends on the support configuration. This end-effect has received very little attention in the literature and the author was unable to find any experimental analysis. Collis and Williams ${ }^{3}$ mention its presence while Mahoney (1956) discusses, qualitatively, some of its aspects from a theoretical viewpoint.

From the above discussion we can infer that, in practice, the free convection radiation temperature corrections will become more valid as the ratio of the wire length to its diameter increases and the ratio of the dimensions of the supports to the wire length decreases.

Forced convection. As with the case of free convection there are some comments pertinent to the application of forced convection data extracted from wind tunnel experiments to the atmosphere. The central issue is the difference in the turbulence regimes. The correlation formulae, $\mathrm{Eq}(9)$ and (10), were derived from steady low turbulence regimes while in the atmosphere there is a comparatively high turbulence regime involving a wide range in the scale and intensity.

The effect of turbulence on the heat transfer from wires has been investigated in the wind tunnel by van der Hegge Zijnen (1958), among others. He discovered that the convective heat transfer is substantially affected only when the ratio of the scale of turbulence to the wire diameter, namely, $L / d$, is less than 10 , with the maximum at $L / d \cong 1.5$; the effect being to increase the transfer to heat. For the wire diameters herein encountered this would imply that the computed temperature corrections are affected only in the smallest scales of turbulence in the atmosphere including themicroscale.

In addition, the heat transfer for $L / d<10$ was found to decrease with decreasing Reynolds number. The minimum Re investigated by van der Hegge Zijnen was 2000 whereas the maximum in this paper is 1000 .

Thus we should expect that the radiative temperature correction based on the mean wind speed in the atmosphere provides a slight overestimation of the real average temperature correction.

\section{APPENDIX A}

\section{Radiation incident on a cylinder}

\section{Downward radiation}

The downward flux of radiant energy incident on a cylinder placed horizontally in the atmosphere can be written

$$
\frac{d E}{d t} \downarrow=\int_{2 \pi} I \downarrow(\theta, \phi)[(S / \pi) \cos \gamma] d \omega,
$$

where $I \downarrow(\theta, \phi)$ is the downward intensity of radiation, $S / \pi$ is the longitudinal cross-sectional area of the 
cylinder, $\gamma$ is the angle between an incoming beam of radiation and a line extending from the beam normal to the cylinder axis, and $d \omega$ is an increment of solid angle.

The term in the brackets represents the effective area of a cylinder perpendicular to all beams of radiation arriving from a given direction.

The radiant energy incident on the end faces of a cylinder has been neglected. The contribution by the end faces to the total energy incident on a cylinder is small when the length/diameter ratio is large.

The geometry for $\mathrm{Eq}(\mathrm{Al})$ is shown in Fig. 3 , where $x$ and $y$ comprise the horizontal coordinates, $z$ is the vertical coordinate, and $\theta$ and $\phi$ are the zenith and azimuth angles, respectively. The $y$ coordinate is parallel to the cylinder axis.

The first step towards the solution of $\mathrm{Eq}(\mathrm{Al})$ is relating $\gamma$ to $\theta$ and $\phi$. From Fig. 3 we see that: $\cos \gamma=d / e$; $\cos \phi=a / b ; \tan \theta=b / c ; d^{2}=a^{2}+c^{2}$; and $e^{2}=c^{2}+b^{2}$. Combining this information we find that

$$
\cos \gamma=\left(1-\sin ^{2} \theta \sin ^{2} \phi\right)^{\frac{1}{2}} .
$$

The next step is to replace $I \downarrow(\theta, \phi)$ by its functional relationship, namely, Eq (2) in Section 2. We repeat it here to provide continuity.

$$
I \downarrow(\theta)=B\left[1-c_{1} \exp \left(-c_{2} \sec ^{\frac{1}{2} \theta} \theta\right) .\right.
$$

Upon substituting Eq (A2) and (A3) into Eq (A1) and recognizing the symmetry of the downward radiation with respect to the zenith, Eq (A1) becomes

$$
\begin{array}{r}
\frac{d E}{d t} \downarrow=4(S / \pi) B\left[\int_{0}^{\pi / 2} \int_{0}^{\pi / 2}\left(1-\sin ^{2} \theta \sin ^{2} \phi\right)^{\frac{1}{2}} \sin \theta d \theta d \phi\right. \\
-c_{1} \int_{0}^{\pi / 2} \int_{0}^{\pi / 2}\left(1-\sin ^{2} \theta \sin ^{2} \phi\right)^{\frac{1}{2}} \sin \theta \\
\left.\quad \times \exp \left(-c_{2} \sec ^{\frac{1}{2} \theta} \theta\right) d \theta d \phi\right] .
\end{array}
$$

Consider the integration of the first member inside the brackets in $\mathrm{Eq}$ (A4). After integration over $\theta$ this member becomes

$$
\int_{0}^{\pi / 2}(1 / 2)\{1-\cos \phi \cot \phi \ln [\cos \phi /(\sin \phi+1)]\} d \phi .
$$

By consulting a table of definite integrals ${ }^{4}$ we find that the above integral is equal to $\pi^{2} / 8$.

The second member inside the brackets in Eq (A4) cannot be integrated in closed form. Therefore, it has been numerically integrated according to the following procedure. Integrating the square root term over $\phi$ we

\footnotetext{
${ }^{4}$ Gröbner, W., and N. Hofreiter, editors, 1958: Integraltafel,
} zweiter teil. Vienna, Springer-Verlag, $204 \mathrm{pp}$.

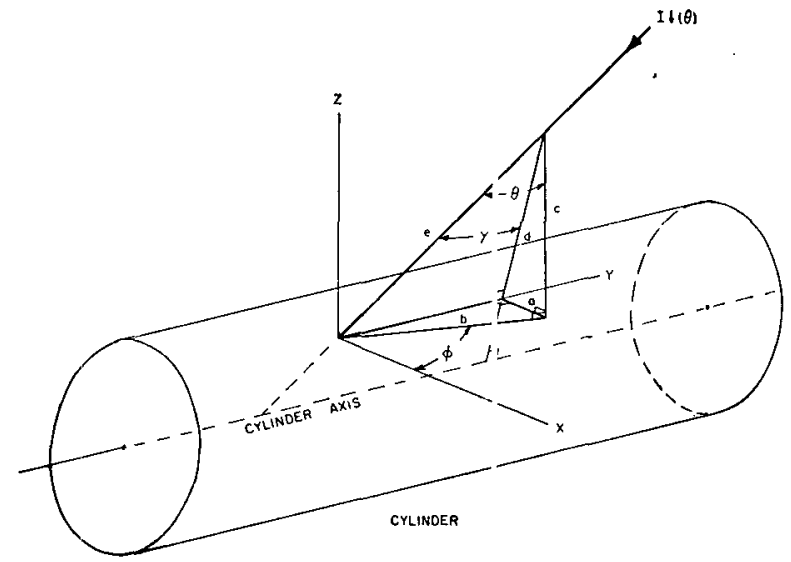

FIG. 3. Geometry of relationship between a beam of radiation incident on a cylinder and the angles $\gamma, \theta$, and $\phi$.

obtain

$$
\int_{0}^{\pi / 2}\left(1-\sin ^{2} \theta \sin ^{2} \phi\right)^{\frac{1}{2}} d \phi=E(\sin \theta, \pi / 2),
$$

where $E(\sin \theta, \pi / 2)$ is the comple te elliptic integral of the second kind. By expanding $E(\sin \theta, \pi / 2)$ in its series form we obtain terms involving even powers of $\sin \theta$.

Consequently, the second member in $\mathrm{Eq}$ (A4) is found to be

$$
\left(c_{1} \pi / 2\right) \int_{0}^{\pi / 2} \sin \theta \exp \left(-c_{2} \sec ^{\frac{1}{2}} \theta_{!}\left(\sum_{i=1}^{n} a_{i} \sin ^{2(i-1)} \theta\right) d \theta .\right.
$$

$a_{i}$ is the coefficient of the $i$ th term in the summation of $n$ terms in the series. Integrating term by term shows that this member can be placed in the form

$$
\left(c_{1} \pi / 2\right) \sum_{j=1}^{n}\left[\sum_{k=1}^{j} b_{j k} E_{4:-1}\left(c_{2}\right)\right] .
$$

$E_{4 k-1}\left(c_{2}\right)$ is the $(4 k-1)$ th exponential integral with argument $c_{2}$ and coefficient $b_{j k}$.

The value of the above memb?r is a function of $c_{1}$ and $c_{2}$.

\section{Upward radiation}

By reversing the direction of the arrow in $\mathrm{Eq}(\mathrm{A} 4)$ and considering only the first member on its righ-hand side including its coefficient we find that after integration

$$
\frac{d E}{d t} \uparrow=\pi^{2}(S / \pi) B / 2,
$$

where we have assumed that $I \uparrow(\theta, \phi)$ is everywhere equal to the horizon intensity $B$ (see $\mathrm{Eq}(3)$ ). 


\section{APPENDIX B}

\section{Ratio of the flux density on a cylinder to the flux density on a plate}

\section{Downward radiation}

The downward flux density on a plate has previously been discussed by Duchon (1963, p. 301). Consequently, the ratio of the flux density on a cylinder to that on a plate can be written

$$
\begin{aligned}
\left(\frac{1}{S / \pi} \frac{d E}{d t} \downarrow\right)_{c} /\left(\frac{1}{A} \frac{d E}{d t} \downarrow\right)_{p} & \\
= & \frac{2\left[\left(\pi^{2} / 8\right)-\left(c_{1} \pi / 2\right) \sum_{j=1}^{n}\left(_{k=1}^{j} b_{j k} E_{4 k-1}\left(c_{2}\right)\right)\right]}{\pi\left[(1 / 2)-2 c_{1} E_{b}\left(c_{2}\right)\right]} .
\end{aligned}
$$

Using the values of $c_{1}$ and $c_{2}$ for each of the 18 sky maps mentioned by Duchon we find that the average value of the 18 ratios computed from $\mathrm{Eq}$ (B1) is 1.62 with all ratios lying within \pm 0.04 of this value. Only five terms in the summation over $n$ were computed. The sixth term is less than 0.5 per cent of the leading term. The exponential integral values were taken from tables computed by Placzek. ${ }^{5}$

s Placzek, G., 1946: The functions $E_{n}(x)=\int_{1}^{\infty} e^{-x u^{-n}} d u$. Natl. Res. Council Canada, Div. Atomic Energy, N. R. C., No. 1547, Chalk River, Ont.

\section{Upward radiation}

The ratio of the upward flux density on a cylinder to that on a plate can be expressed as follows.

$$
\left(\frac{1}{S / \pi} \frac{d E}{d t} \uparrow\right)_{c} /\left(\frac{1}{A} \frac{d E}{d t} \uparrow\right)_{p}=\pi / 2 .
$$

$\mathrm{Eq}$ (B2) follows from $\mathrm{Eq}$ (A5) and the fact that the upward flux density on a plate is $\pi B$.

\section{REFERENCES}

Baldwin, L. V., V. A. Sandborn and J. C. Laurence, 1960: Heat transfer from transverse and yawed cylinders in continuum, slip, and free molecular air flows. Trans. $\triangle S M E$, Series $C$, 82, 77-86.

Collis, D. C., and M. J. Williams, 1959: Two-timensional convection from heated wires at low Reynolds numbers. $J$. fluid Mech., 6, 357-384.

Duchon, C. E., 1963: The infrared radiation temperature correction for spherical temperature sensors. $J$. appl. Meteor., 2, 298-305.

Gröber, H., 1961 : Fundamentals of heat transfer. New York, McGraw-Hill, $527 \mathrm{pp}$.

van der Hegge Zijnen, B. G., 1957 : Modified cor:elation formulae for the heat transfers by natural and by firced convection from horizontal cylinders. Appl. Sci. Res. A, 6, 129-140.

- 1958: Heat transfer from horizontal cylind air flow. A ppl. Sci. Res. A, 7, 205-223.

Mahoney, J. J., 1956: Heat transfer at small Cirashof numbers. Proc. Roy. Soc. $A, 238,412-423$.

McAdams, W. H., 1954: Heal transmission. New York, McGrawHill, 532 pp. 